\title{
Event Management for Social Service Website Using Ruby on Rails: BTO Event View Application Developed Implementing Collaborative Technique
}

\author{
Younten Tshering, Sai Preetham Kamishetty, Hekmatullah Sarwarzadah
}

\begin{abstract}
Usually, event management is the process of creating and managing an event in a project organization. In most cases, effective management is required to make an event public and productive. For this reason, an efficient tool of managing events through an online solution helps an organization to get better outreach and efficient management. The objective of the solution is to support organizations to have event management, tracking, and making the activities known to the general public. The application was intended for Bhutan Toilet Organization (BTO), a non-profitable organization located in Bhutan, which provides clean toilet facilities during public events organized. Currently, BTO is using a manual-based system in managing and facilitating the events and activities organized by them and their clubs who contribute to achieving the mission of BTO. By having the Web-based Event Management System (BTOEventView), it will let the authorized user create and update the activities or events and the users and public will be more alert and aware of the existence of the events or activities held under BTO. This project aimed to develop a website, which easily allows managing the event management process and eases the efforts of an event organizer and the clients. The user interface was implemented by using the MVC design pattern in the Ruby on Rails framework and developed applying collaborative technique which is pair-programming with three programmers. Moreover, to make the website mobile compatible Bootstrap framework was used. The application offers a platform to create events, view events, or manage the events based on privileges provided to users. User management was also created to manage the system users and web page contents.
\end{abstract}

Index Terms - Event Management, MVC, Pair-programming, Ruby on Rails, and Web-based or Website.

\section{INTRODUCTION}

Event management is the process of creating and managing an event in a project organization. Events are managed in an enormous range and different ways are used to manage the events. Efficient and effective management is essential to make any kind of an event successful. For this reason, an efficient tool of managing events through an online solution helps an organization to get better outreach and efficient management.

Younten Tshering, Associate Lecturer, Royal University of Bhutan, Master's in Computer Science with A Specialization in Software Engineering

Sai Preetham Kamishetty, Master's in Computer Science with A Specialization in Software Engineering, Asian Institute of Technology

Hekmatullah Sarwarzadah, Master's in information management, Asian Institute of Technology
Bhutan Toilet Organization (BTO) is a non-profitable organization located in Bhutan, which provides clean toilet facilities during public events organized across the country since most of the event locations do not have sufficient toilet facilities to cater to the huge numbers of people. Currently, BTO is using a manual-based system in managing and helping the events and activities organized by them and their clubs who contribute to achieving the mission of BTO. By having the Web-based Event Management System (BTOEventView), it lets the authorized user create and update the activities or events and the users and public will be more alert and aware of the existence of the events or activities held in BTO.

The application was developed and named as BTOEventView, and it is a web application. It has been developed using the Ruby on Rails Framework with the pair-programming concept with three programmers. Likewise, Bootstrap is used for responsive building and others are also used such as CSS, HTML5, and JavaScript. An online event management solution is directly proportional to its members who hit the application and register themselves [1]. BTOEventView has been designed in an organizational context and made the complex process easy. The objective of the solution is to automate/manage several types of events online.

Basically, this system will help the College Club Ambassadors, Project manager, and the administrators for that particular organization. The College Club Ambassadors, Project manager, and other staff will be updated with the events of the particular organization, alerted with the existence of the events and announcements of the events. This system is not only applicable for the College Club Ambassadors, Project manager, and other BTO staff, but also to the public outside the BTO. The public will be able to know about the upcoming events in BTO and events carried out by the club and BTO.

The effective use of the system might help the organization to ensure the involvement of the College Club Ambassadors. This system will help to keep the public alert and aware of the events in BTO. Their contribution can also be encouraged to ensure full support for certain events. The involvement or membership from the public can also be encouraged to enhance the good image of BTO as they have a more systematic system for this kind of situation. Based on the above-stated descriptions, for BTO we have used the "Rapid Application Development (RAD)" methodology to complete the objectives. This approach provides the process, collaborative techniques, and tools to deliver the project in an 
efficient way. The aim of the project was to develop event management and automate the process of event management.

\section{RELATED WORK}

As per [3], Web-based systems have been implemented by other international universities. The students overseas are more flexible with the updated technology. Few international universities have been identified using the Wireless Application Protocol (WAP) based system. The National University of Singapore applied the WAP, and all event details were retrieved anytime on a single WAP page. According to [4], the author mentioned such a system will give an easy, faster, and comfortable way to access the online system for the application associated with their university. Hence, a WAP-based Event Management System was proposed in managing the events information in University Teknologi Petronas (UTP).

From paper [2], there is no proper management of the events in University Teknologi Petronas (UTP). Everything was paper-based, and event management has not existed. Students and staff were alerted of the upcoming events when they read pamphlets or the newsletter posted on the wall at the hostel and academic buildings. In reality, there were a lot of events that has been organized by the UTP staff and students. As justified before, some organizations have organized a lot of events that required involvement from the students. Occasionally, students use to not get the information about those events because they were not alert about that event. The problems given showed the difficulties to the students and other people involved in keeping up to date with the events at the UTP and creating awareness of their participation in certain events. For this problem, the new system, the WAP-Based Event Management System is developed as the solution.

From chapter [6], we can see the process of creating web applications based on the Ruby on Rails framework where this technology-facilitated and accelerated creation of web applications. At present most businesses offer web applications using PHP language. It lets accomplish most of the necessary functionality, but the time spent on implementation is often much longer than in Ruby. Rails make common CRUD operations like these much easier. The realistic element includes the formation of a system supporting the operation based on the Ruby on Rails framework. The system development enables coordination of the work between the employees by allocating them tasks to be performed and indicating the time they have for a given assignment. The paper focuses on each step and the principle of application development which shows how to control data flow in the Model-view-controller (MVC).

Therefore, to develop the BTOEventView we used Ruby on Rails and implemented MVC architecture which is a software design pattern. With BTOEventView, using the above concept discussed, College Club Ambassadors can be easily updated with the information about events when browsing the system by using a PC and mobile phone. Even the administrator of the system can update their events and College Club Ambassadors and Project Manager can do the same without worrying about the time and place.

\section{METHODOLOGY}

As per [1], systems development life cycle (SDLC) is a conceptual model used in project management that explains the stages involved in an information system development project, from an early planning phase which includes feasibility study till the completion of the application. For developing the system, the developer followed modern approaches, as discussed in [6] software lifecycle actions were adopted to support and develop the software system. As per paper [6], this project followed the UML approach, which has improved the process of knowing the system and architecture's layout to be strong to manage concurrency. With the design, we implemented the concept of multi-page applications (MPAs) and single-page applications (SPAs). For MPAs we have to concentrate on the architecture and technology utilized on the server-side. For SPAs, we look at the architectures of both the frontend and backend systems and their interactions.

For the BTOEventView, we implemented the Rapid Application Development (RAD) keeping in mind that time and getting end users involved to verify the product quality. Since the limitation of development time, the traditional development process cannot be applied as one of the methodologies to complete this project. Because of that, RAD methodology had been chosen as the backbone of the development process. The idea behind RAD is that end users can give better feedback when assessing a system, as opposed to working rigorously with documentation. One of the main advantages is that RAD-based development has resulted in less rejection when the application is placed into production.

RAD has analysis, design, build, and test phases into a series of short, iterative development cycles. This has several distinct advantages over the traditional development model. Iteration allows for effectiveness and self-correction. Studies have shown that human beings rarely accomplish a difficult task correctly the first time. Nevertheless, people are good at making an acceptable beginning and then making many minor refinements and enhancements.

\section{A. Planning phase}

In the planning phase, problem identification was important. A clear objective was drawn from the problem that had been identified. Multiple solutions were researched with a literature review on event management. In this development, the problem statement and the scope of research had been clearly stated in the introduction. During this phase, the project timeline and milestone had also been built according to the duration that had been allocated by the stakeholder (supervisor). After the planning, a comprehensive explanation of the application and its features is given below covering requirement analyzing, architecture designing, and the implementations. The use case diagram of the system can be seen in figure 1.

\section{B. Design phase}

The design phase gets the input from the requirements collection in the planning phase. Design parts describe the application features in detail and normally contain functional or dataflow diagrams, interface diagrams, UML diagrams, 
and an entity-relationship diagram.

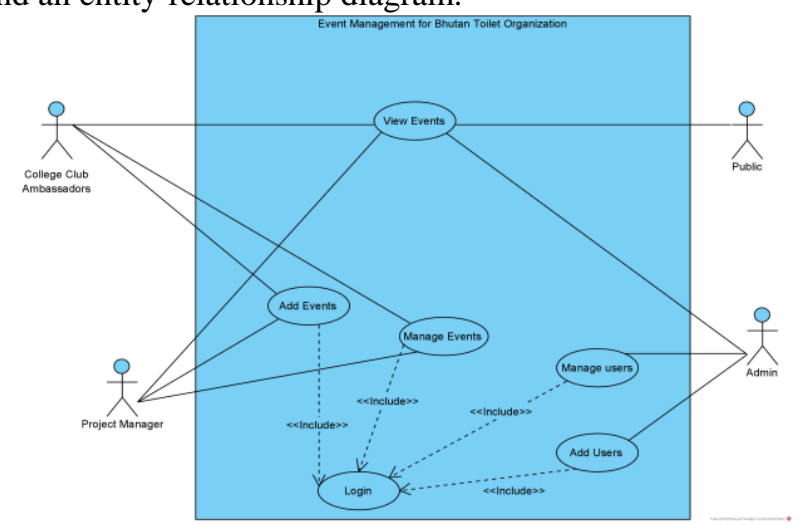

Figure 1. Use Case Diagram

The database consists of 4 tables shown in figure 2. The user table represents the users of BTOEventView. It maintains 3 different roles i.e., admin, project manager, and college club ambassador. It has other information such as first_name and last_name. It also has "is_active" which is used to ban/unban user account. The event table has event name, description, time, duration. It has a foreign key that is a user id from the user table. Events have a many-to-many relationship with the User table through the Register table. That is, a user has many events, and an event has many users. Register table is a join table for users and events. It joins them using user_id (User) and event_id (Event). A location table is used to store the location description.

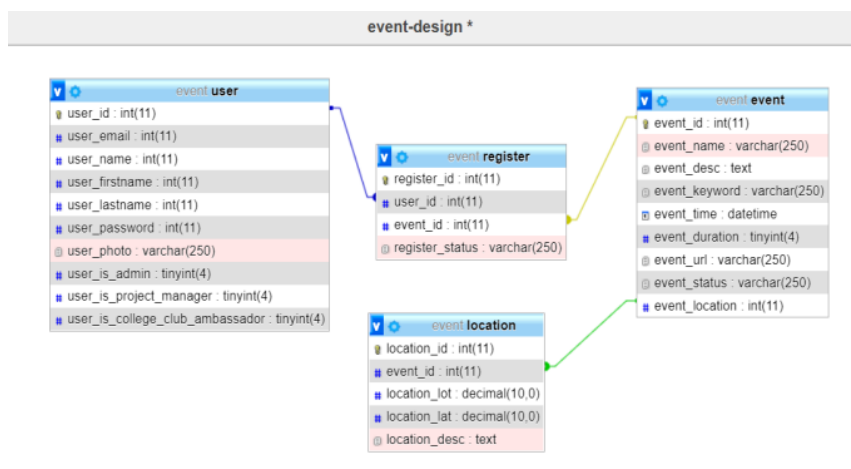

Figure 2. Data Model / Database design

It is a project where we planned on making our website pretty simple. As the project will be displaying the events organized by the BTO and its associations. We planned on having the link to this project along with BTO website. On clicking the link, the users can view the events in a table format with their organizer and below it, we have displayed all details that are required to be displayed. We have only one interface where the operations can be performed by the Project Manager and College Club Ambassadors.

The other workflow of our design is explained in the figure 3 and 4 where it shows the flow of user registration and content management page. For the project, there is only one major interface that will be displaying the events held or in progress by the organization to the public. Therefore, the Project Manager, College Club Ambassadors, and Admin is allowed to do the CRUD operations and others can only view the information.

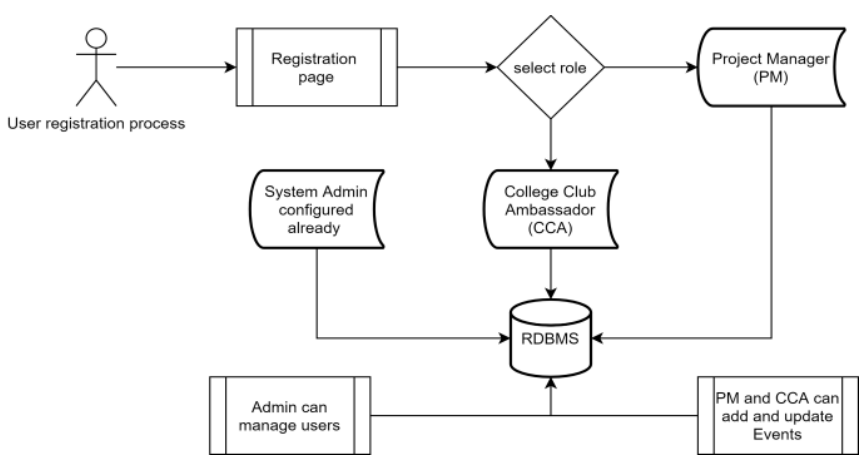

Figure 3. User registration and management flow

Figure 4 shows the content data model is managed after the user registered is retrieved from the database for admin, then admin checks the list of users registered and performs the action to enable or disable. If the user is approved by the admin, then the user becomes a project manager or college club ambassador. Once approved, the user can perform a CRUD operation for events. The main interface retrieves the information about the event from the database and the general user or public can view the list of events created by the approved user.

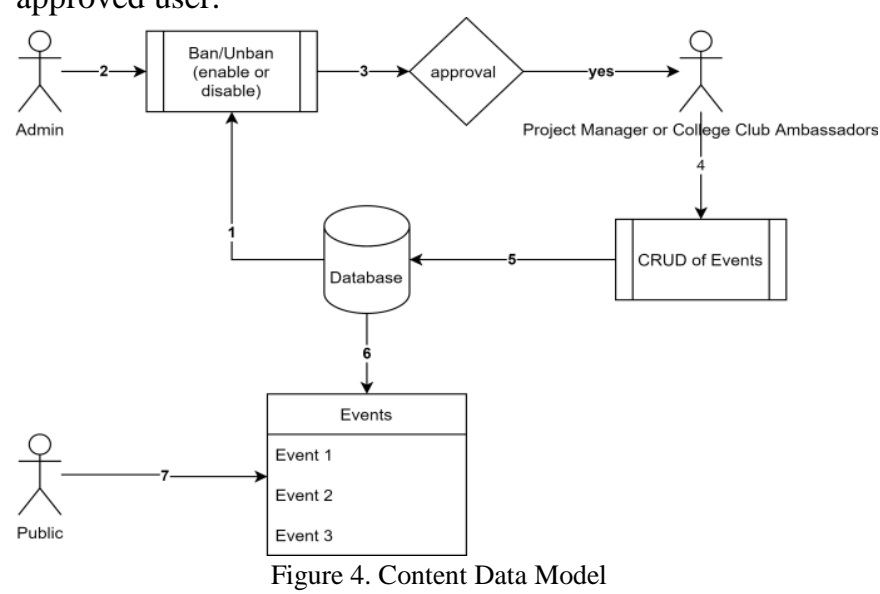

\section{Development phase}

The development phase takes input from design components defined in the design document. The output of the development phase includes a fully functional set of software that satisfies the requirements and design elements earlier documented. The development phase of the project can be seen in Figure 5

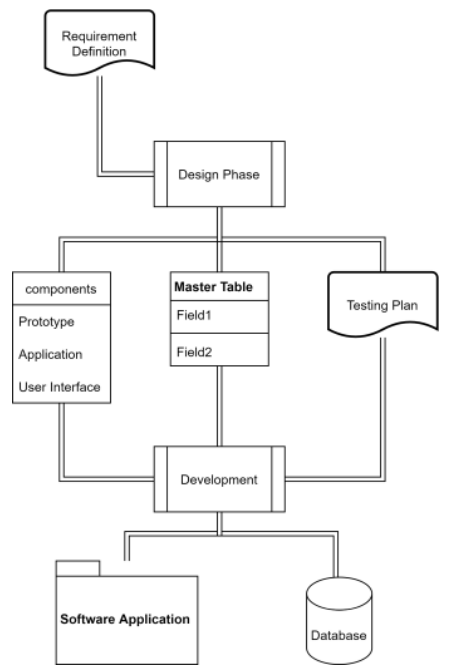

Figure 5. Development phase - input and output 


\section{Event Management for Social Service Website Using Ruby on Rails: BTO Event View Application Developed Implementing Collaborative Technique}

While developing the system, the approach applied, or implemented was the collaborative technique which is pair-programming. For this project, we were three in numbers, we applied the same technique of pair-programming with three programmers working together online via sharing screen. Pair programming is a collaborative technique to do some tasks together with efficiency and effectiveness. This kind of method is used as an agile practice. In pair programming we need to swap roles as driver and navigator, respect each other, and should have some common knowledge while doing the work.

Learning outcome from developing system in pair-programming with three members, as a driver, we need to follow the navigator instruction and we should not feel embarrassed or hesitate while making mistake. We just need to focus on solving a problem and if we go wrong then the navigator will be there to correct or verify the answer. As a navigator, we need to let the driver start the task and try to understand how the driver proceeds with the work. After understanding, we need to check whether we are on track and give suggestions based on what we think is best compared to the driver's methods. As a navigator, we need to observe and cross-check the work done.

We felt that pair programming is much better in terms of efficiency and quality to enable ourselves to collaborate. In pair programming with three members, we are more careful and confident with the solution that we establish since all agree on a common aspect. If we, do it alone, we might not be sure whether the solution is accurate or not, and sometimes we do not know how to start but in pair programming, we do not have to worry about it.

During the development of the BTOEventView, as mentioned earlier we used the model-view-controller (MVC) pattern since it gives way to separate logic, the data model, and the way in which those data are viewed. This helps in abstraction and separation of concerns which lead to less coupling and high cohesion. MVC was mainly used due to two reasons: firstly, the business logic is decoupled from the presentation logic and the secondary reason is the business logic can be tested outside of a Web server. The main thing at the end is that MVC provides modularity, separation of concerns, and abstractions that enhance the maintainability of the application. Figure 6 shows how MVC is working in Ruby from the book [7].

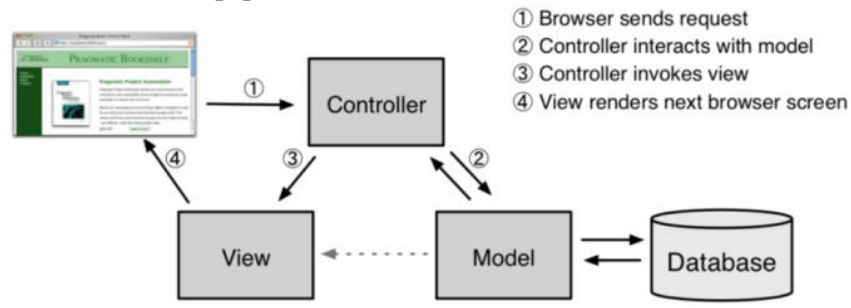

Figure 6. MVC in Ruby on Rails framework

The data model encapsulates the application-specific data used by the system. View modules receive model data and format it for display. Controller sections respond to user actions, execute operations on the data model, and accordingly pick views for the user.

\section{Testing phase}

To keep quality high during web application development, it is known that a rigorous developer testing methodology is the single most effective technique for delivering high-quality web applications on time and within budget. During the integration and test phase, all test cases were run to verify the correctness and completeness of the software using tools like Cucumber for acceptance testing and Rubocop \& SimpleCov for unit testing to ensure complete coverage of our source codebase. Overall "red-green-refactor" development method was simple to implement as 1 . add a test, 2 . tests should be executed and checked for failure, 3. write a few codes, 4. run tests then repeat from step 3. until all tests pass, 5. refactor source code, and 6. repeat from step 1 .

Testing is the most important part of the software development process, to guarantee the correct layout and functionality of an application. Furthermore, the developed process has given needed information about different scenarios where the application may have a chance to collide. The application has been greatly tested during all phases of the development. Tests have been completed for each functional module, as well as the application. For BTOEventView, the developer and stakeholder work together to write automated acceptance tests that express what the stakeholder expects the software to do.

Finally, some significant suggestions for making testing jobs to be effective for the development of applications could be to test early, test often, and test automatically. Thus, we can work on the following:

1) Get a production build up and running on a test server early in the development and give stakeholders access to the server.

2) Create an automated regression test suite and keep adding to it as defects are found.

3) Use a continuous integration tool such as GitLab CI to automatically download the latest build, run the test suite, and generate quality metric reports.

4) Add appropriate tests as we code and ensure the regression test suite passes before every commit.

\section{RESULTS}

At the initial step, various assessment methods have been done to find out the satisfaction of users for the Event Management System. The first approach that has been done was an observation, where the current system has been examined to identify the problems and the solution for those problems. Then implementation process helped foresee the unexpected pitfalls and manage each step in a well-defined manner. With a good implementation plan, we could define the general goals to achieve the system requirements and the timeframe to complete the plan where the project succeeded.

The application incorporates the news updates which the users can access to get more information related to various awareness programs conducted by organizations across the world in improving the awareness among the people. As you visit the website, the sign-in and sign-up options are displayed at the top-right. When you click on Sign up, the form is retrieved where you can enter your details. As the user, you need to select the designation from (Student Coordinator, Project Manager, Viewer) to use the application. 


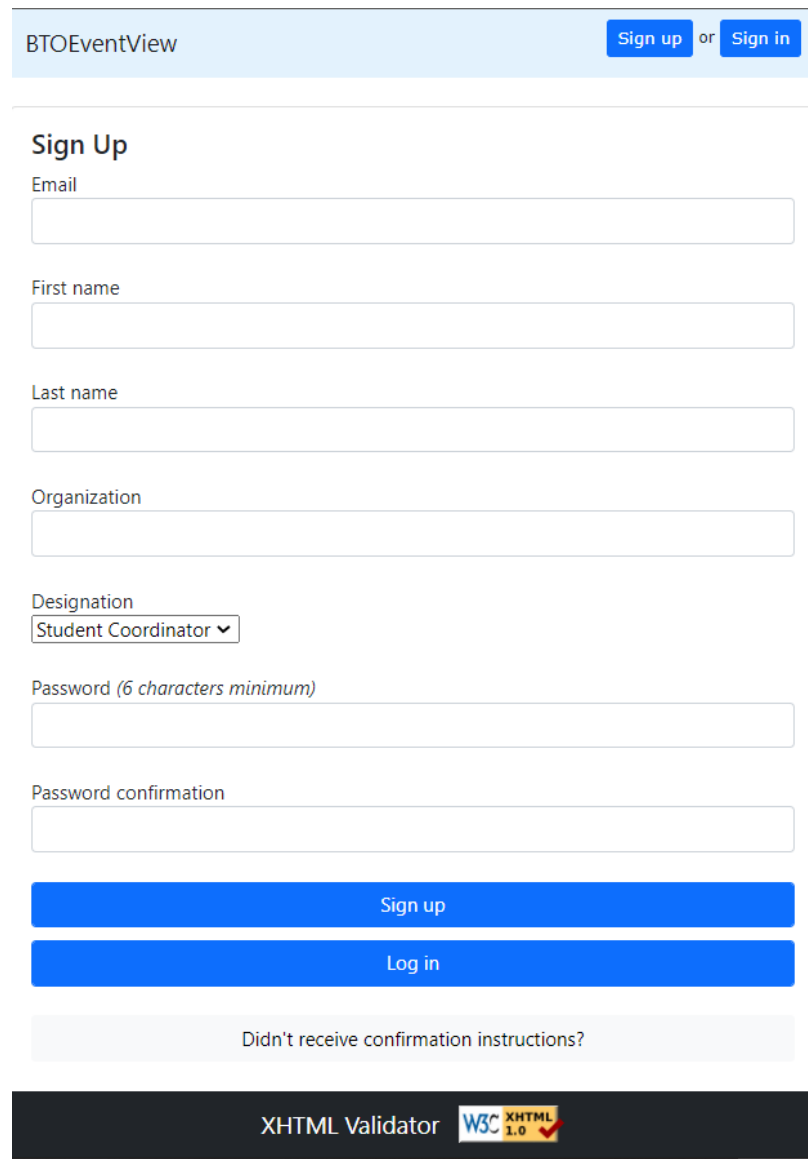

Figure 7. Sign up Form

For Sign in, we need to enter your email id and password and click on Log in. Firstly, if you log in as Admin into the website then you will have all privileges. Then, the admin can go to the Admin Page for user management. As Admin you can have access to the pages such as Recently Registered Users, User Stats, Ban Users, and Events.

\section{Admin \\ \begin{tabular}{ll|l|l}
\hline Recently Registered Users & User Stats & Ban Users & Events
\end{tabular}}

Figure 8. Admin privileges

In Recently Registered Users, as Admin, we can view the list of recently registered users information with First Name, Last Name, Organization, Designation, State of the user, Email Id, Account Creation Time. In User Stats, as Admin, we can view the total number of users registered on the website per month and year.

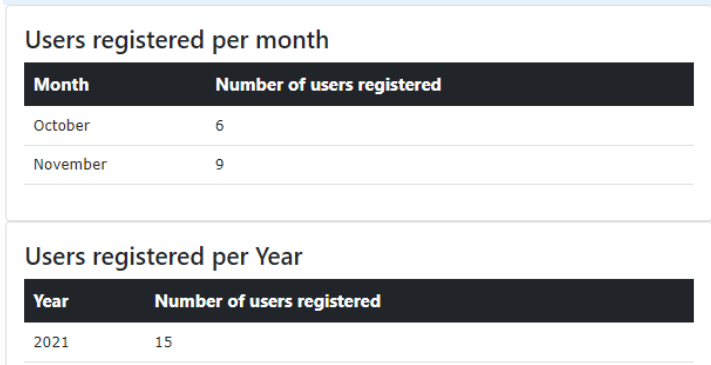

Figure 9. User Statistics

In Ban Users, as Admin, we can view the User Details such as Name, Email, Designation, State of the User, and get options to Ban/Unban and also change the Role of the User.
Admin will be able to manage the users by Banning or Unbanning them. Admin can also change the role of users. There are two types of roles given to the users as 1 . The member who is a regular user and can view the events available and 2. The manager who can view and create, manage the events such as the Project Manager, Student Coordinator.

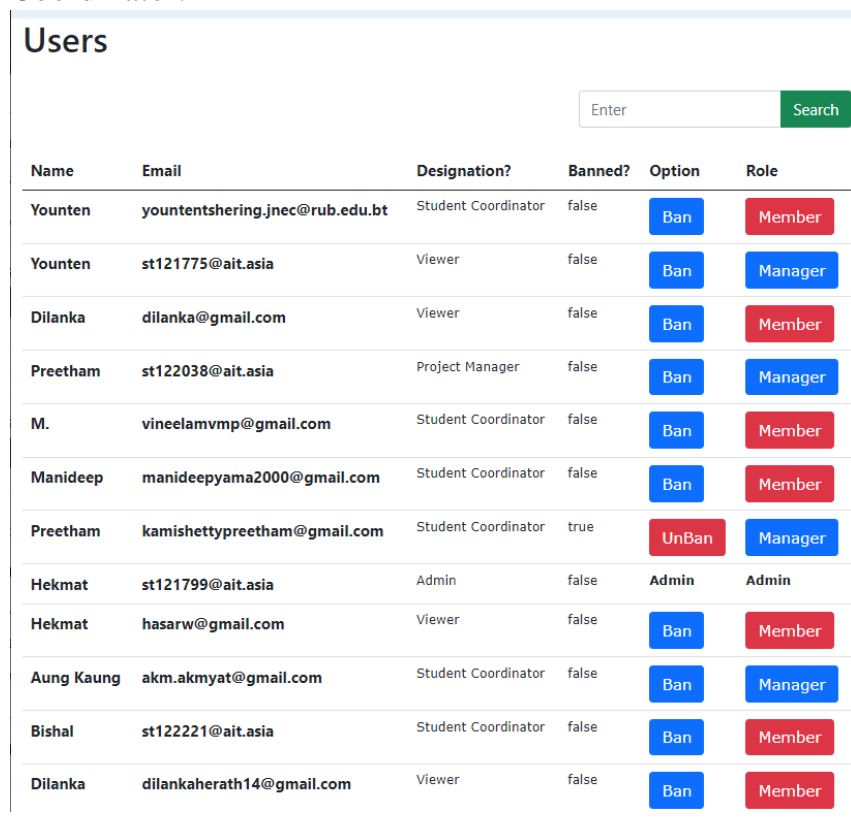

Figure 10. User Management

In Events, as Admin, we can view the events available and edit or destroy (delete) them. When you click on the show, the event details are shown in detail. When the admin clicks on Edit, the page for editing event data is retrieved. When you click on a new event, the Adding New Event page is displayed where we can enter new event details.

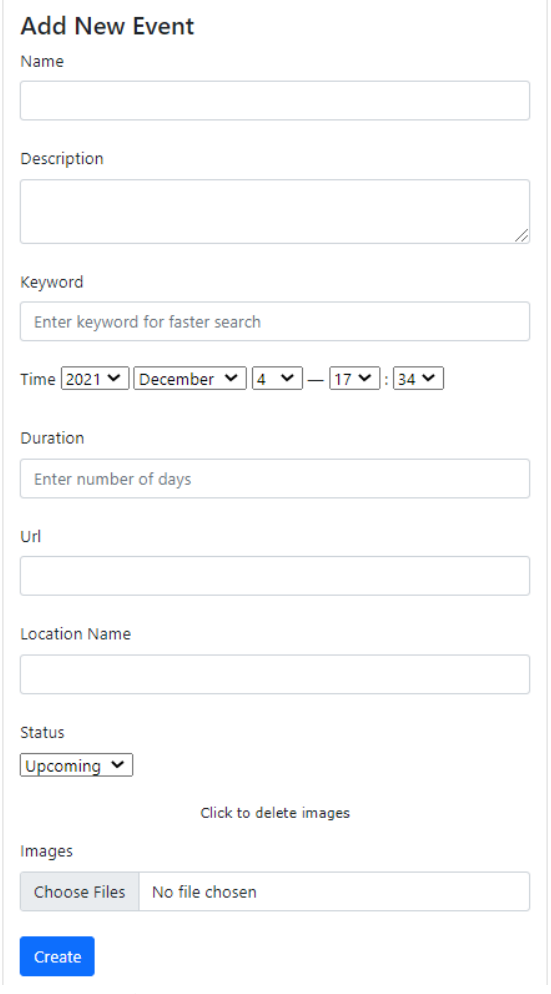

Figure 11. New Event Form

As Project Manager and Student Coordinator, they need to 


\section{Event Management for Social Service Website Using Ruby on Rails: BTO Event View Application Developed Implementing Collaborative Technique}

log in as same and will be able to see the events page to know the event information. When they click on the Events page, they will be able to view, edit and destroy the events. They also have the privilege to create new events. When they click on the show, event details are shown. As Viewer, when they Log in, they can see the Events interface with a list of upcoming events, ongoing events, and completed events. As a Viewer, if they want to view the details of the event then have to click on the details option.

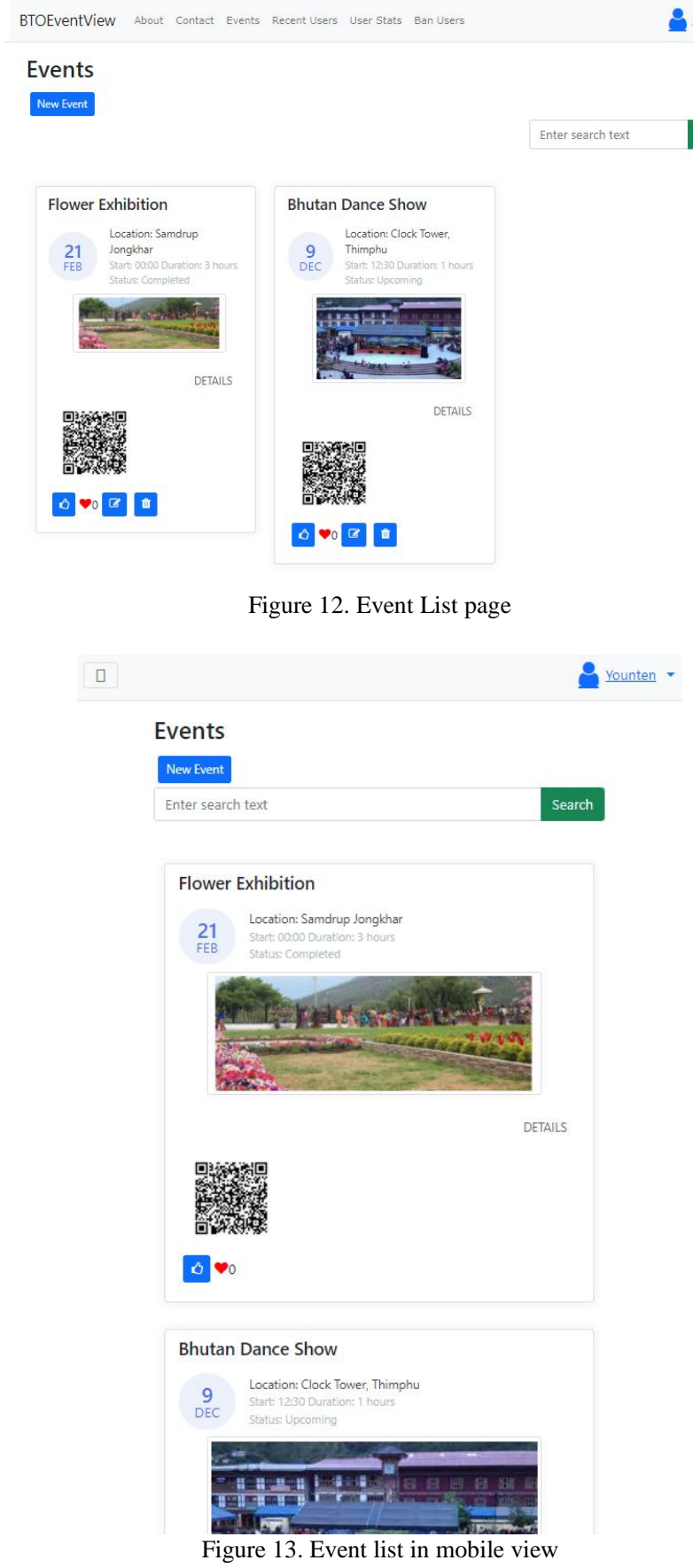

The Application provides a generated $\mathrm{QR}$ code whenever a new event is created. This QR code can be scanned to retrieve the details of a particular event. It helps in increasing the promotion of the event in a fast and efficient way providing detailed information. The application also has search functionality to retrieve relevant events based on Name, Location, and Keyword. There are also filters that help in getting the events based on status and duration to help the users.

\section{Flower Exhibition}

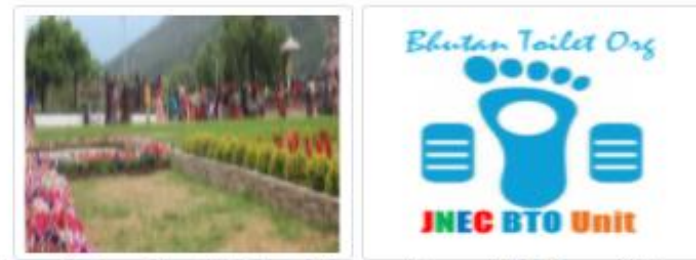

Description: The 5th Royal Bhutan Flower Exhibition will be held at Samdrup Jongkhar. The exhibition is open to the public and promises to be as amazing as always.

Keyword: Samdrup

Time: 2019-02-21 00:00:00 UTC

Duration: 3

Url: https://www.jnec.edu.bt/en/jnec-clean-toilet-initiatives/ Location name: Samdrup Jongkhar

Status: Completed

created_by: st121799@ait.asia

Edit

\section{Back}

Figure 14. Event Detail page after clicking show option

Acceptance tests must be defined from the customer's point of view. The test outcome can be found as shown in figure 15 where UATs (User Acceptance Testing) are passed as users should be able to get event list, search event, get the details, and like the events.

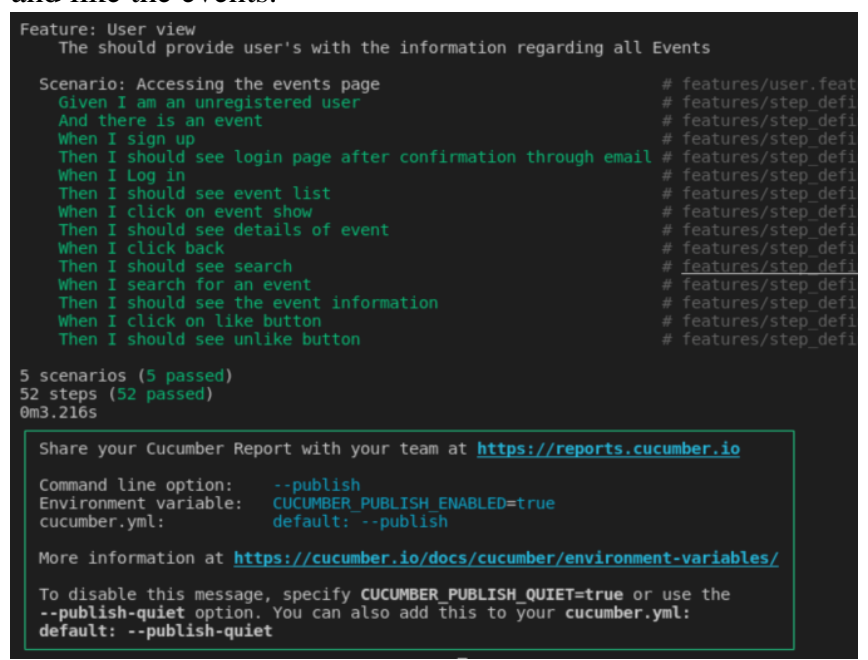

Figure 15. Cucumber Scenario passed

Robot Framework is Python-based to assess automation. This framework helps write an object-page model in keyword-driven methodology. We can test web app access by writing the script in VS Code with robot file extension and running it will show the test case pass or fail. The test case implemented in the robot framework where the test data is in a simple format. As soon as Robot Framework is executed, it runs test cases and then creates logs files and reports. To check the automation, the test case created was the user will open the website, then login into the system as a viewer, click 
the show button, view the event details then click the back button and click the like button (if the user liked it) and then logout from the system and at the end close the browser after work done.

Once we have the test case, we can run the test and check the test case pass or fail. Figure 16 shows that the test case developed was passed and the automation worked completely for that scenario. After the test is completed, it generates output, log, and report files to confirm the output in detail and if there are any fail cases in the test then a screenshot is also produced to know which test failed.

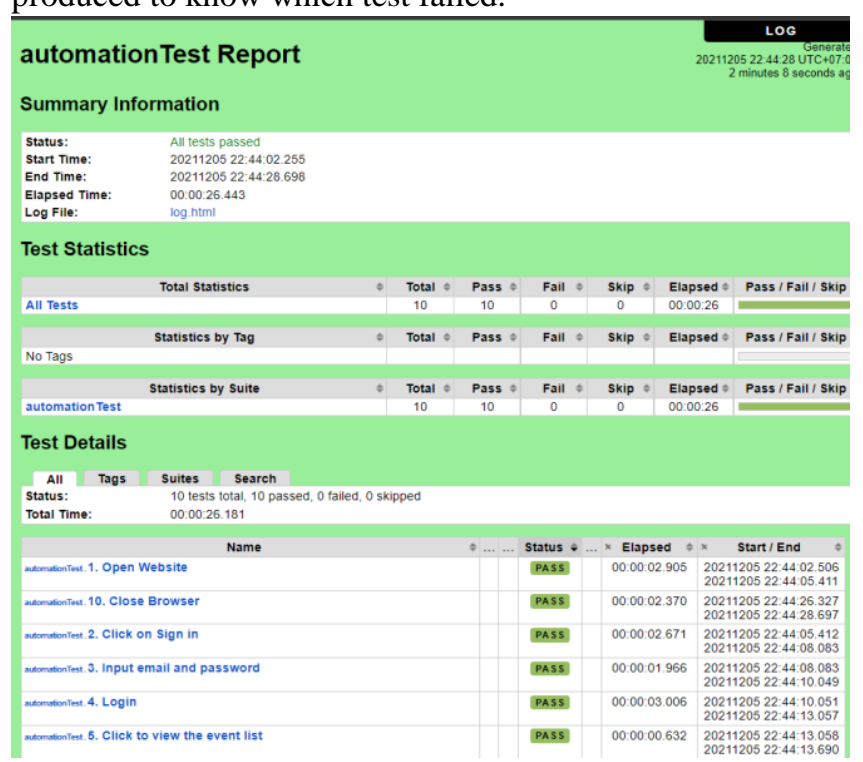

Figure 16. Test case Report

To test the multiple access or request was tested using JMeter. A test plan was created which is the central execution unit of a test. The test configures elements of a test plan such as Thread Group, Samplers, and Listeners. We configured 3000 threads in 1 loop, making it 3000 threads or users access the website. Our application was competent to handle the multiple access over 3000 and we tested till 5000.

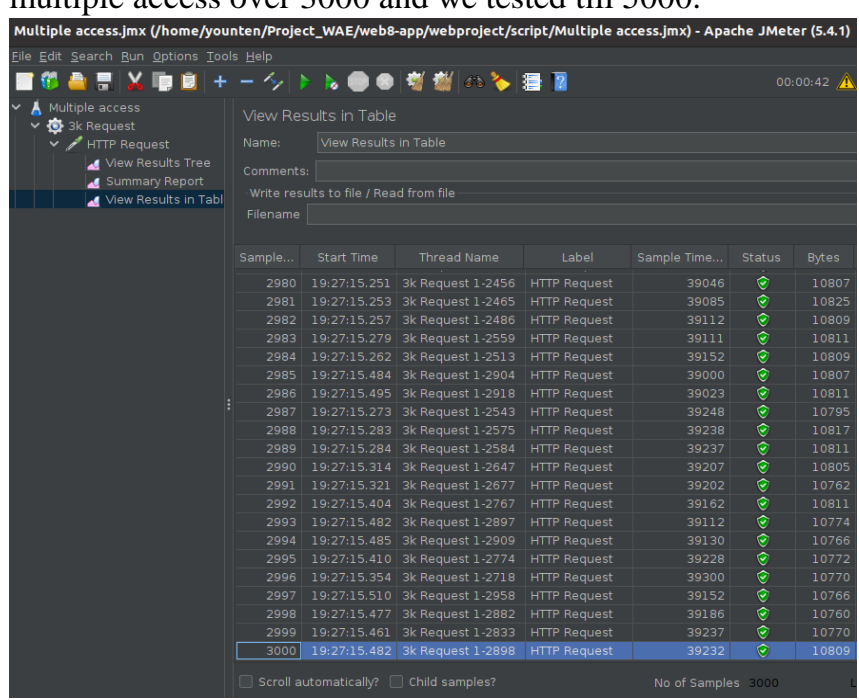

Figure 17. Result of multiple user access

To check more details on the system design document, user guide or manual to follow the system and source code of the project can be found in the link given below:

https://github.com/Younten-Tshering/Projects and_related works/tree/main/BTOEventView\%20Application

\section{DISCUSSION}

The application developed was to be dynamic website where it requires more sophisticated means of persistence. Thus, we used the relational model since it is good for frequent updates to structured data. We know that RDBMS pass the ACID test where Atomicity (transactions are all or none), Consistency (the database moves from one reliable state to another), Isolation (partially completed transactions are not visible to any other user), Durability (completed transactions must survive power outages). After deciding to use an RDBMS, the following steps were useful i) developing the data model, ii) determining the set of legal transactions on the data model, iii) designing the page flow, and iv) implementing each page. While designing and implementing the project, it was noticed that application will be used for reading more than writing. Therefore, if it becomes a big data application then NoSQL databases (Schemaless databases) can be more common to use such as document databases, key-value databases, column family databases, and graph databases.

For the development process, we can consider collaborative technique such as pair-programming with three programmers working online if partners are willing to work and idea matches with one another. If the partner is not willing to share the idea or not participate actively in work, then it will be a wastage of time or will be equivalent to working alone. In our point of view, pair programming should be practiced in every work since it helps in bringing productivity and there is more learning since we get to learn and share ideas. We think pair programming with three will not work if the partners are from a different educational background or do not possess the same knowledge on the problem to solve.

Regarding time consumption with pair-programming with three members working online, we observed that it does not make many differences since working individually requires verification, reviewing some references, and thinking on the procedure. In pair programming with three, it takes time in terms of scheduling meetings and discussion, but it reduces the time for verification and review. For this project, pair programming with three showed positive time taken for solving the problem since all three had a common understanding of the development process, way of working was matching and were fully ready for the task or problem to be solved. The switching role was interesting since others get to learn how other people work and gather additional knowledge.

To check the structural design and performance, testing is the best way to check rather than using hypothetical claims. With an aggregate report from JMeter, it was found that there is an $8.57 \%$ error for multiple access with 3000 threads in 42 seconds but with a hold target rate of 10 minutes for the same 3000 threads, it showed $0 \%$ of error.

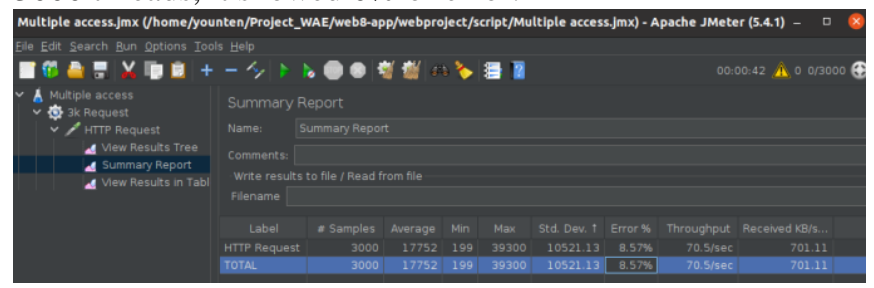

Figure 18. Aggregate report 


\section{Event Management for Social Service Website Using Ruby on Rails: BTO Event View Application Developed Implementing Collaborative Technique}

Testing and developing together does help and with the test-first methodology, we can write test cases before writing the code that makes the test pass. Writing test cases before coding has helped in coding since it makes it easier to think about the design before starting coding, it helps to detect poorly specified requirements and defects sooner than it would otherwise. Therefore, finding defects early as possible can be time-saving and energy-saving.

\section{CONCLUSION}

As information systems grow in power, it is likely that soon information systems will be the source and resource of information as a single model. Users will see such a system as just a technology that gives access to the information of events via the Internet directly. Event management includes a great sense and even better organization. This includes managing the events through different online features. The development was implemented in a very reasonable way using a collaborative technique such as pair-programming with three programmers. Future improvements and implementation are provided to the shareholders and suggested the organization for development. The design took time but still, it was in the project schedule. The objectives of the project were achieved, and the shareholders were convinced. One of the main challenges while doing this project was to set up a server. After spending some time and from the discussion in the forums we did manage to resolve the server issues. Later, another obstacle came when the team had to combine the modules. It was a bit tricky to make sure that each module is implemented correctly.

Currently, the BTOEventView can be viewed by any user and only authorized users can update the events. The reason why the system cannot let the public create or update events is because of the existing system scope. As for future work, a general user or public can participate in the event after registering or signing up. Even the deployment problem can be solved since we worked on the proxy server and the system can be completely functioning. In supplement, for future improvement, the BTOEventView can be developed with all the objectives achieved, whereby the public is more alert and updated with all the events information held or organized by the organization. This might encourage public participation in the events. In conclusion, by developing this BTOEventView, the problems encountered by Bhutan Toilet Organization to maintain the events organized by college clubs will be eliminated. They can obtain updated events information on their website.

\section{ACKNOWLEDGMENT}

We would like to take this opportunity to thank Professor Matthew N. Dailey, as the project supervisor and for his courage, support, patient, help, and guidance throughout this project. We would like to thank Mr. Karma Tenzin, Student Service Officer who has been our stakeholder in developing the system and was involved in all development processes. We also would like to thank my beloved parents, who have faith and support that we can do it.

Finally, thank you to everyone who is involved directly or indirectly in providing a great contribution to this project.

Upon completing this work, we would like to convey our gratitude to ourselves. We are grateful to the individuals who have contributed their ideas, points of view, encouragement, and support for this project.

\section{REFERENCES}

[1] Ashfaq, A. (2014). Event Management System: EventCart.

[2] Bidin, M. S. A. (2007). WAP-Based UTP Event Management System.

[3] McComb, Dave, 2004, How Does WAP Application Can Be Embedded To The Other Applications, USA, Elsevier

[4] Azilah Binti Madulan @ Alias, 2005, WAP Application with Event Responses System Using Java, Degree Thesis, University Teknologi Petronas, Malaysia

[5] Tshering, Younten \& Tamrakar, Suyogya \& Gontia, Shubhangini \& Baral, Smrity. (2021). IoT-based Platform with Big Data using Apache Kylin: Air Quality Monitoring System (AQMS). World Journal of Research and Review. Volume-13. 13-20. 10.31871/WJRR.13.3.12.

[6] Luczak P., Poniszewska-Maranda A., Karovič V. (2021) The Process of Creating Web Applications in Ruby on Rails. In: Kryvinska N. Greguš M. (eds) Developments in Information \& Knowledge Management for Business Applications. Studies in Systems, Decision, and Control, vol 330. Springer, Cham. https://doi.org/10.1007/978-3-030-62151-3_9

[7] Ruby, S., Copeland, D. B., \& Thomas, D. (2020). Agile Web Development with Rails 6. Pragmatic bookshelf.

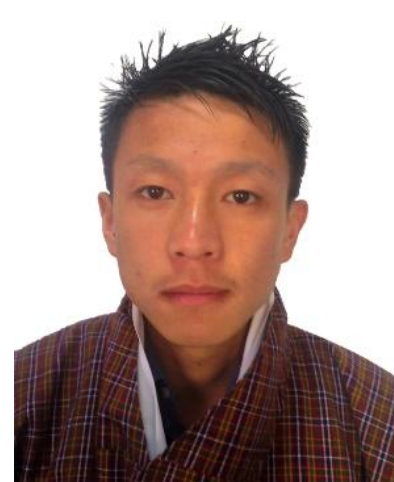

interoperability in e-government.
Younten Tshering is an Associate Lecturer in the Department of Information Technology, Jigme Namgyel Engineering College, Royal University of Bhutan. $\mathrm{He}$ has completed B.Sc. in Computer Science (Honors) and obtained an academic excellence certificate. He is currently pursuing his master's degree in computer science with a Specialization in Software Engineering at the Asian Institute of Technology. Research interest is in software development \& management, semantic web technology, and

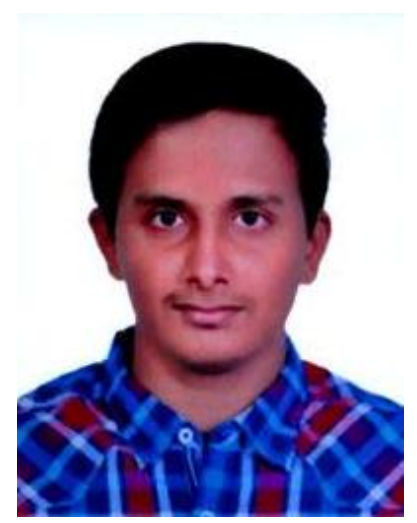

Sai Preetham Kamishetty is a computer science engineer, currently pursuing his master's degree in Computer Science with a Specialization in Software Engineering at the Asian Institute of Technology. He worked as a Research and Development intern in the area of cloud computing and cyber security. Research interest is in Cloud Computing, Machine Learning, and Software Development. 


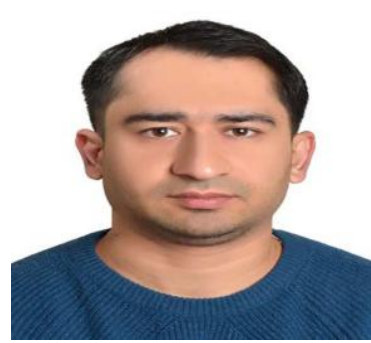

Hekmatullah Sarwarzadah is a former Data Analyst at WorldVision International in Afghanistan. He is a master's degree student of Information Management at the Asian Institute of technology. He has a bachelor's degree in Computer Science with software engineering. Research interest is in database system and programming. 